\title{
Case Report: Evaluation of Gait Performance in Hemipelvectomy Amputation While Walking With a Prosthesis
}

\author{
Mohammad Taghi Karimi ${ }^{1}$, Mostafa Kamali ${ }^{\text {* }}$ (D) \\ 1. Department of Orthotics and Prosthetics, School of Rehabilitation Sciences, Isfahan University of Medical Sciences, Isfahan, Iran.
}

\begin{tabular}{|l|l|l}
\hline $\begin{array}{c}\text { Use your devic to scan } \\
\text { and read the article online }\end{array}$ \\
Crtation Karimi MT, Kamali M. Evaluation of Gait Performance in Hemipelvectomy Amputation While Walking With a \\
Prosthesis. Physical Treatments. 2018; 8(1):55-61. http://dx.doi.org/10.32598/ptj.8.1.55
\end{tabular}

(c) (1) (8)

Article info:

Received: 23 Aug 2017

Accepted: 01 Feb 2018

Available Online: 01 Apr 2018

Keywords:

Hemipelvectomy amputation, Canadian prosthesis, Gait analysis

\begin{abstract}
A B S T R A C T
Purpose: Hemipelvectomy amputation is a surgical procedure in which the lower limb and part of the pelvis are removed. Although few studies are available on the performance of individuals with hip disarticulation while walking, there is no study on gait analysis of hemipelvectomy subjects. Therefore, this study aimed to evaluate the gait and stability of an individual with hemipelvectomy amputation.
\end{abstract}

Methods: An individual with hemipelvectomy amputation on the right side participated in this study. He used a Canadian prosthesis with single axis ankle joint, 3R21 knee joint and 7E7 hip joint for more than 10 years. The kinetic and kinematic parameters were collected by a motion analysis system and a Kistler force plate.

Results: There was a significant difference between knee, hip and ankle motion ranges and their related time periods on the sound and prosthesis sides. The stability of the subject was better in the anteroposterior direction, than the mediolateral direction. Results revealed a significant asymmetry between the sound and prosthesis sides.

Conclusion: The obtained results suggested a significant asymmetry between the kinetic and kinematic performance of the sound and prosthesis sides, which may be due to lack of muscular power and alignment of prosthesis components.

\footnotetext{
* Corresponding Author:

Mostafa Kamali, MA.

Address: Department of Orthotics and Prosthetics, School of Rehabilitation Sciences, Isfahan University of Medical Sciences, Isfahan, Iran.

Phone: +98 (913) 3505258

E-mail:mostafa_kamali@rehab.mui.ac.ir
} 


\section{Highlights}

- Hemipelvectomy is an operation in which the lower limb and part of the pelvis are removed.

- We conducted a study on gait analysis of a hemipelvectomy subject.

- The results indicate a significant asymmetry between the kinetic and kinematic performance of the sound and prosthesis sides.

- This difference might be due to lack of muscular power and or alignment of prosthesis components.

\section{Plain Language Summary}

In hemipelvectomy amputation the lower limb and part of the pelvis are removed. Because, there is no study on gait analysis of hemipelvectomy subjects, we aimed to evaluate the gait and stability of an individual with hemipelvectomy amputation. He used a Canadian prosthesis with single axis ankle joint, for more than 10 years. According to our results, there was a significant difference between knee, hip and ankle motion ranges and their related time periods on the sound and prosthesis sides. The stability of the subject was better in the anteroposterior direction, than the mediolateral direction. The obtained results suggested a significant asymmetry between the kinetic and kinematic performance of the sound and prosthesis sides, which may be due to lack of muscular power and alignment of prosthesis components.

\section{Introduction}

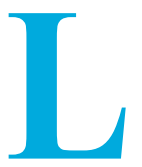

ower limb amputation occurs as a result of traumas, vascular diseases, cancers and so on. The incidence of amputation varies from 2.8 to 43.9 per 100000 individuals, of which 0.5 to 3 is related to hip-level amputation or above $[1,2]$. The main causes of amputation at this level are vascular problems, malignancy, and tumors [1-3]. The hip joint amputees lose their ability to stand and walk efficiently (without crutches and walkers), thus they use certain prostheses [2-4].

Different types of prostheses have been designed to restore standing and walking abilities in amputees at the hip joint surface and hemipelvectomy [4, 5]. However, several problems including high energy expenditure for walking, slow walking, inappropriate walking style in terms of aesthetics, and limited motion range of lower limb joints, are associated with the usage of prostheses [6-10]. As a result of limited users of this type of prosthesis, few studies are available in this regard. In addition, research studies are scare on influential kinetic and kinematic parameters in users of Canadian prostheses $[6,7,9]$. Only one study evaluated the effect of applied force on the prosthesis while walking in a hip joint amputee [8].

The average speed of walking with the prosthesis in a hip joint amputee differed from 0.83 to $1.31 \mathrm{~m} / \mathrm{s}$ [7].
Moreover, this person had a step length between 0.65 and $0.96 \mathrm{~m}$ which was significantly different from the normal population $[7,11]$. The energy consumption of the amputee was also approximately 2 times higher than the normal population [11]. Unlike hip amputation, hemipelvectomy amputation is a surgical procedure in which the entire lower limb and part of the pelvis are removed. Therefore, it seems that the performance of an amputee is different than people with hip disarticulation, due to the higher level of amputation. According to the literature, no study assessed the ability of people with hemipelvectomy amputation. Therefore, this study is the first one to evaluate the amputees' performance in this situation.

\section{Materials and Methods}

A person with a hemipelvectomy amputation of the right side participated in this study. He used a form of Canadian prosthesis with a multiaxial wrist joint, 3R21 knee joint and 7E7 hip joint for over 5 years. The weight, height and age of the subject were $75 \mathrm{~kg}, 1.75$ $\mathrm{m}$ and $39 \mathrm{y}$, respectively.

The study measured parameters were spatial-temporal walking parameters (walking speed, cadence, step length, stance phase), torque applied on lower limb joints, the movements of the lower limbs and trunk joints in 3 directions, and the force applied to the legs during walking. In addition, the balance of the subject 
was measured while standing on a force plate with open- and closed-eyes for 1 minute. Balance was evaluated through the following formulas:

Equation 1:

$\operatorname{COPEAP}(m m)=X_{\max }-X_{\min }$

Equation 2:

COPEML $(\mathrm{mm})=Y_{\max }-Y_{\min }$

Equation 3:

$\operatorname{PLAP}(\mathrm{mm})=\sum_{n}^{n-1} \sqrt{\left(x_{i+1}-x_{i}\right)^{2}}$

Equation 4:

$\operatorname{PLML}(\mathrm{mm})=\sum_{n}^{n-1} \sqrt{\left(y_{i+1}-y_{i}\right)^{2}}$

Equation 5:

$\operatorname{VAP}(\mathrm{mm} / \mathrm{min})=\frac{\sum_{n}^{n-1} \sqrt{\left(x_{i+1}-x_{i}\right)^{2}}}{t}$

Equation 6:

$\operatorname{VML}(\mathrm{mm} / \mathrm{min})=\frac{\sum_{n}^{n-1} \sqrt{\left(y_{i+1}-y_{i}\right)^{2}}}{t}$

COPEAP, COPEML, PLAP, PLML, VAP, VML were respectively the range of pressure center changes on the anterior-posterior plane, the range of pressure center of internal-external plane, path length of the pressure center on the anterior-posterior plane, path length of pressure center on external-internal plane, the velocity of the pressure center on the anterior-posterior plane, and the velocity of the pressure center on the internal-external plane. Distribution of variables was evaluated by Shapiro-Wilk test at a significance level of 0.05 . Parametric tests were used for final analysis, due to normal distribution of the variables. The difference between sound and prosthetic side was evaluated through 2-sample t-test at the significance level of 0.05 .

\section{Study methods}

Kinetic and kinematic evaluation were conducted at the Musculoskeletal Research Center of Isfahan University of Medical Sciences, Isfahan City, Iran by using a Swiss made 7 high-speed 3D motion capture solution (Qualisys motion tracking system) and a Kistler force plate (Kistler Model 9285, Quartz Force, USA). The obtained data were analyzed using Visual3D software (C-Motion Inc., USA). Therefore, the force and torque applied to

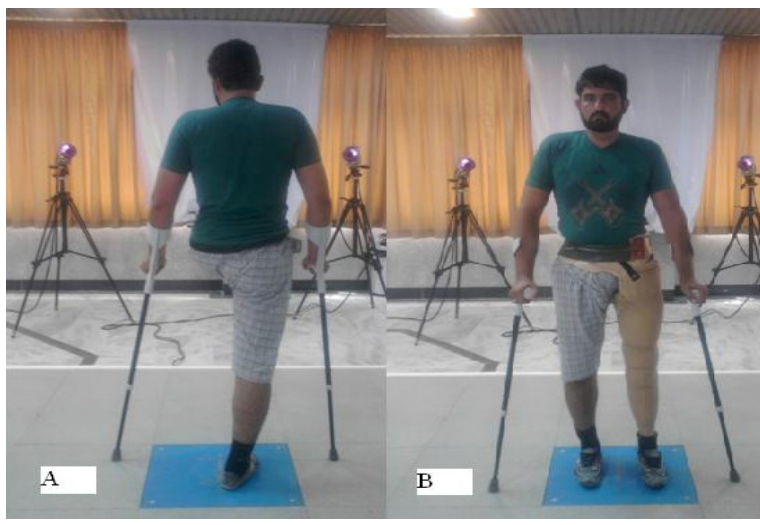

PHYSICAL TREA TMENTS

Figure 1. Person with a hemipelvectomy amputation

A. Without a prosthesis; B. with Canadian prosthesis

various joints from raw data were calculated. Force plate frequency and the cameras were set to run at $120 \mathrm{~Hz}$.

The participant was asked to stand and walk in the laboratory. Markers used in this test were round with 14 $\mathrm{mm}$ of diameter, covered with light reflectors in order to be identified by cameras. Markers were installed on the legs, hips, and trunk, according to the marker installation protocol used at the Department of Biomedical Engineering, University of Strathclyde, Glasgow, Scotland, the United Kingdom.

Sixteen markers were placed on the Anterior Superior Iliac Spine (ASIS), the Posterior Superior Iliac Spine (PSIS), internal and external ankles, heads of the first and fifth metatarsal, and the left and right large trochanters. In addition, 4 markers were placed on semicircular clusters and connected to the anterior side of the leg and thigh by flexible straps. The subject was requested to walk at safe speed in the laboratory (the subject walked with a crutch in order to maintain balance and avoid falling). Tests were repeated 10 times while the subject was walking with a Canadian prosthesis. Independent samples $t$ test with a significance level of 0.05 was used as the final analysis. Statistical tests were performed in SPSS.

\section{Results}

The motion range of the wrists, knees, and thighs on all 3 planes are listed in Table 1. As specified in Table 1, Mean motion ranges of the wrist joint on the sound side of the body on sagittal, coronal, and transverse planes were equal to $34.18,17.45$, and 13.92 , respectively, compared to 4.96, 2.95 and 6.88 on the prosthetic side. There is a significant difference between the sound and prosthetics sides regarding knee joint motion ranges 
Table 1. Comparison of the motion range of hip, knee, and wrist joints in 3 planes for the sound and prosthetic sides

\begin{tabular}{cccccccccc}
\hline Parameter & $\begin{array}{c}\text { Flexion/ } \\
\text { Extension of } \\
\text { the Wrist }\end{array}$ & $\begin{array}{c}\text { Inversion/ } \\
\text { Aversion of } \\
\text { the Wrist }\end{array}$ & $\begin{array}{c}\text { Wrist } \\
\text { Rotation }\end{array}$ & $\begin{array}{c}\text { Flexion/ } \\
\text { Extension of } \\
\text { the Knee }\end{array}$ & $\begin{array}{c}\text { Abduction/ } \\
\text { Adduction of } \\
\text { the Knee }\end{array}$ & $\begin{array}{c}\text { Knee Rotation } \\
\text { Extension of } \\
\text { the Hip }\end{array}$ & $\begin{array}{c}\text { Abduction/ } \\
\text { adduction of } \\
\text { the Hip }\end{array}$ & $\begin{array}{c}\text { Hip } \\
\text { Rotation }\end{array}$ \\
\hline $\begin{array}{c}\text { Sound side } \\
\text { Prosthesis } \\
\text { side }\end{array}$ & $34.2 \pm 18.12$ & $17.1 \pm 45.28$ & $13.2 \pm 92.14$ & $46.6 \pm 95.52$ & $3 \pm 15.25$ & $19.2 \pm 71.12$ & $40.7 \pm 3.16$ & $7.1 \pm 61.20$ & $9.1 \pm 46.12$ \\
P & $4.0 \pm 96.12$ & $2.0 \pm 95.19$ & $6.1 \pm 88.02$ & $24.3 \pm 98.85$ & $7.1 \pm 66.28$ & $4.0 \pm 59.15$ & $27.3 \pm 33.65$ & $6.0 \pm 9.92$ & -- \\
\hline
\end{tabular}

Data are presented as Mean \pm SD.

PHYSICAL TREA $\mid$ MENTS

Table 2. Comparison of pelvis and thorax kinematic parameters in sound and amputated sides

\begin{tabular}{|c|c|c|c|c|c|c|c|c|c|c|c|}
\hline 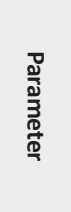 & 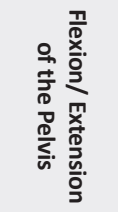 & 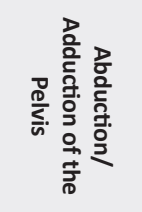 & 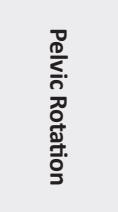 & 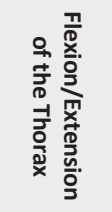 & 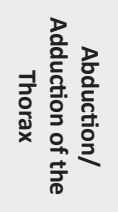 & 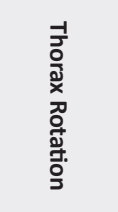 & 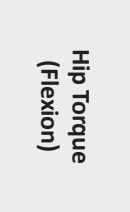 & 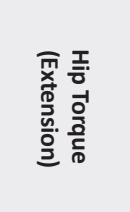 & 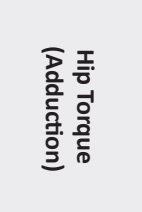 & 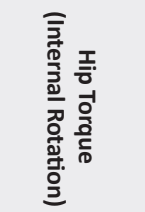 & 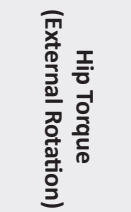 \\
\hline $\begin{array}{l}\tilde{0} \\
\frac{\bar{a}}{0} \\
\frac{u}{0} \\
\frac{0}{D}\end{array}$ & $3.0 \pm 34.15$ & $18.0 \pm 5.08$ & $6.1 \pm 37.05$ & 3.01 & $2.0 \pm 59.06$ & $4.0 \pm 9.02$ & $0.0 \pm 32.001$ & $0.0 \pm 811.06$ & $1.0 \pm 21.01$ & $0.0 \pm 39.0006$ & $0.0 \pm 75.002$ \\
\hline 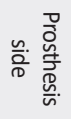 & $2.0 \pm 89.01$ & $14.1 \pm 318.02$ & $5.1 \pm 61.00$ & $11.2 \pm 6.02$ & $3.1 \pm 22$ & $4.0 \pm 84.99$ & $0.0 \pm 187.01$ & $0.0 \pm 31.004$ & $0.0 \pm 358.007$ & $0.0 \pm 34.002$ & $0.0 \pm 46.02$ \\
\hline o & 0.1 & $<0.001$ & 0.24 & 0.035 & 0.017 & 0.39 & 0.01 & $<0.001$ & $<0.001$ & 0.33 & 0.056 \\
\hline
\end{tabular}

$(\mathrm{P}<0.05)$. The Mean range of hip joint motion on the sound and prosthetic sides were 40.3 and 27.33 degrees, respectively, which indicates a significant difference between the 2 sides $(\mathrm{P}=0.001)$. The Mean motion range of abduction/adduction of hip joint on the sound side was 7.61 degrees and it was 6.9 degrees in the prosthetic leg, which does not suggest a statistically significant difference between the 2 sides $(\mathrm{P}>0.05)$.
Table 2 presents the pelvis and thorax kinematic parameters on the sound and amputated sides. As per Table 2 , there is no difference between the pelvic motion range on the sound side and the amputated side, on the sagittal and transverse plates. The Mean range of forward bend and side bend were 14.02 and 2.59 degrees on the sound side and 11.06 and 3.22 degrees on the prosthetic side, respectively $(\mathrm{P}<0.05)$. We also explored torque applied on the hip joint. Mean range of flexor torque on the hip

Table 3. Comparison of the torque applied to the wrist joint and the force transmitted on both sides

\begin{tabular}{|c|c|c|c|c|c|c|c|c|c|c|c|}
\hline 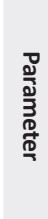 & 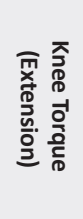 & 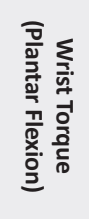 & 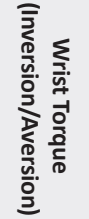 & 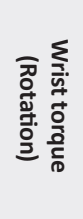 & 齐 & 余 & श & $\mathbb{N}$ & 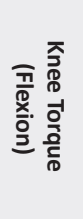 & 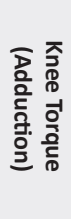 & 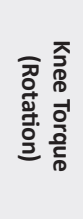 \\
\hline
\end{tabular}

\begin{tabular}{|c|c|c|c|c|c|c|c|c|c|c|c|}
\hline $\begin{array}{l}\tilde{c} \\
\frac{0}{0} \\
\frac{a}{\frac{a}{a}}\end{array}$ & $0.0 \pm 147.07$ & $1.0 \pm 432.002$ & $0.0 \pm 528.003$ & $0.0 \pm 242.01$ & $6 \pm 51.12$ & $17 \pm 110.12$ & $9 \pm 27.23$ & $865.56 \pm 8.22$ & $0.0 \pm 44.15$ & $0.0 \pm 32.0012$ & $0.0 \pm 09.01$ \\
\hline 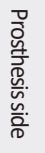 & $0.0 \pm 202.003$ & $0.37 \pm 0.01$ & $0.0 \pm 120.116$ & - & $9.1 \pm 16.25$ & $15.1 \pm 31.23$ & $1 \pm 2819.66$ & $248.22 \pm 8.22$ & $0.0 \pm 126.12$ & $0.1 \pm 0.0007$ & $0.05 \pm 0.1$ \\
\hline$\nabla$ & $<0.001$ & $<0.001$ & $<0.001$ & $<0.001$ & $<0.001$ & $<0.001$ & $<0.001$ & $<0.001$ & 0.003 & 0.008 & 0.001 \\
\hline
\end{tabular}


Table 4. Mean \pm SD scores of stability parameters in open- and closed-eyes mode

\begin{tabular}{|c|c|c|}
\hline \multirow{2}{*}{ Parameter } & \multicolumn{2}{|c|}{ Mean $\pm S D$} \\
\hline & Open-Eyes & Closed-Eyes \\
\hline Path Length of $X(\mathrm{MM})$ & $425.506 \pm 67.97$ & $412.37 \pm 216.149$ \\
\hline Path length of $Y(\mathrm{~mm})$ & $510.47 \pm 313.93$ & $403.5 \pm 636.37$ \\
\hline Excursion of the pressure center on the $\mathrm{X}$ plane $(\mathrm{mm})$ & $5.056 \pm 1.874$ & $3.19 \pm 0.54$ \\
\hline Excursion of pressure center on $\mathrm{Y}$ plane $(\mathrm{mm})$ & $227.4 \pm 0.54$ & $1.568 \pm 0.447$ \\
\hline The speed of the pressure center on the $X$ plane $(\mathrm{mm} / \mathrm{s})$ & $885.135 \pm 13.95$ & $824.74 \pm 433.298$ \\
\hline Speed of the pressure center of on $\mathrm{Y}$ plane $(\mathrm{mm} / \mathrm{s})$ & $1020.149 \pm 627.86$ & $807.10 \pm 273.74$ \\
\hline
\end{tabular}

joint on the sound and prosthetic sides were 0.32 and $0.186 \mathrm{Nm} /$ body weight, respectively $(\mathrm{P}=0.01, \mathrm{P}=0.01)$.

Hip joint extensor torque on the sound side was 2 times more than the prosthetic side. Although there was a significant difference between the hip joint adductor torque on both sides, the difference between the Mean rage of torque transferred from the knee was not significant. As specified in the Table, knee torque on the prosthetic side was significantly different from that of the sound side. Figures 1, 2 and 3 show the movements of the hip, knee, and joint of the wrist on the sound and prosthetic sides.

Table 3 presents the Mean range of torque, applied to the wrist joint and the force transmitted from both sides. The Mean vertical impact on the left leg was equal to 865.8 $\mathrm{N}$, compared to $248.8 \mathrm{~N}$ on the prosthetic side $(\mathrm{P}<0.001)$. The first and second peaks of posterior-anterior force on the sound side were 51 and $110 \mathrm{~N}$, compared to 9.16 and $5.31 \mathrm{~N}$ on the prosthetic side, respectively $(\mathrm{P}<0.001)$. The torque applied to the wrist joint on the amputated side was significantly higher than that of the sound feet and the difference between them was significant.

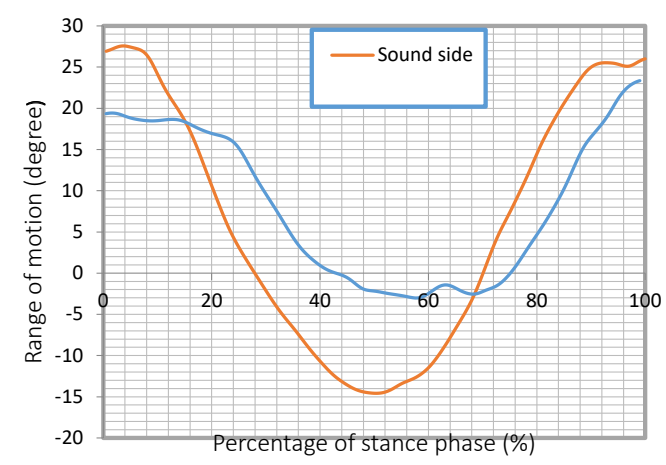

PHYSICAL TREA $\$ MENTS

Figure 2. Flexion/extension of the hip joint in the sound and prosthetic sides

\section{Discussion}

Hemipelvectomy amputation is a surgical procedure in which the lower limbs and a part of the pelvis are removed. Limited studies have been conducted on the performance of amputees in respect of the hip surface while walking [1]. Therefore, there is no study on the analysis of individuals with hemipelvectomy. As a result, this case report aimed to evaluate the walking and balance of people with this type of amputation.

As per Table 1, hip, knee, and wrist joints kinematics are significantly different between the sound and prosthetic sides. Hip joint motion range on the sound side on the sagittal plane was 40.3 degrees, compared to 27.33 degrees for the prosthetic feet. The main reason to this is the hip position on the prosthetic side which is placed on the anterior part of the socket [5]. Flexion and extension motion range of the knee joint were also less than those of the sound side which can be due to prosthetic components alignment $[5,2]$.

The main strategy used by amputees to control the movements of prosthetic joints was changing the position of the center of gravity, relative to the artificial joint center. The alignment of the prosthesis is adjusted in such a way that the line of weight is always in the front of the knee joint and be-

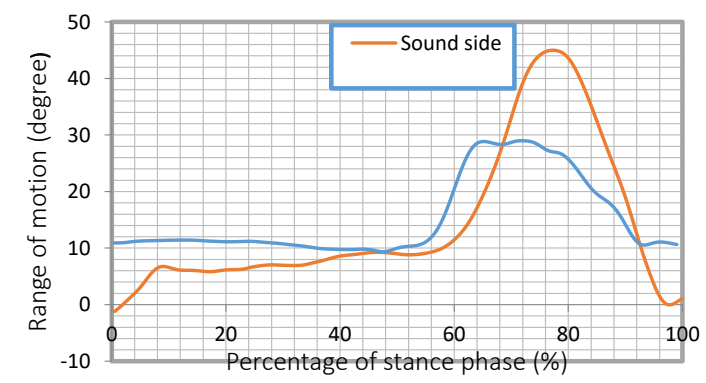

PHYSICAL TREA MENTS

Figure 3. Extension-flexion in the sound and prosthetic sides 


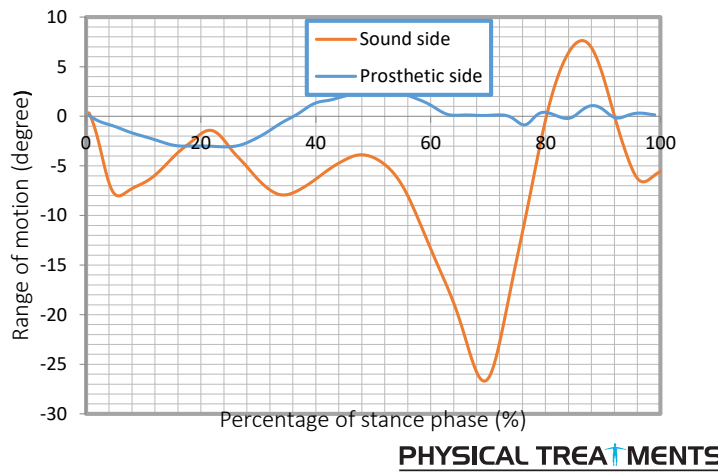

Figure 4. Plantar/ dorsiflexion range of motion of wrists on the sound and prosthetic sides

hind the hip joint. Therefore, hip and knee joints are always maintained in the extension mode during the stance phase [7, 2]. There was no major differences between pelvic flexionextension and rotation on both sides regarding the pelvic motion. The main reason for this issue is the special design of the socket which covers the pelvic [7, 4]. Therefore, the pelvic motion range is significantly limited on both sides and the upper edge of the socket comes up close to the trunk.

Another parameter investigated in this study was the torque applied to the hip joint. Hip joint torque on the prosthetic side was significantly less than that of the sound side. The main reason for this may be related to the force transmitted from the legs on the prosthetic side. The Mean scores of flexion/extension torque and hip joint abduction in a sound person were equal to 0.4 to $0.98,0.75$ to 0.98 and $0.97 \mathrm{Nm} /$ body weight, respectively [11], which is roughly the same as torque applied to the sound side in this study.

Limited limb movement range on the prosthesis side can be another reason. In a study conducted by Nietert et al. flexion torque and hip joint abduction were 1.1 and 0.98 , respectively [8], which were higher than the results of this study. The main reason to this difference might be using crutch in this study. The study participant used crutch to improve his balance while walking and standing. A portion of body weight is applied to the crutch which affects the torque on to the joints and the components of the prosthesis.

The force transmitted through both legs is essentially different. In other words, the magnitude of the breaking and progression forces are significantly different on both sides. However, these 2 forces are almost the same in a normal person, which indicates the gait symmetry [11]. According to the present study, not only the mean score has been significantly reduced, but also the first and second peak rates were not the same, on the prosthetic side.
This difference can be due to the strength of prosthetic side muscle and use of a cane.

The Mean scores of balance equilibrium parameters on the open- and closed-eyes modes are presented in Table 4. Motion Mean \pm SD range of Center of Pressure (COP) of the body on the internal-external and anterior-posterior planes in the open-eyes mode were $5.056 \pm 1.87 \mathrm{~mm}$ and $4.0 \pm 22.54 \mathrm{~mm}$, respectively. COP fluctuation Mean $\pm \mathrm{SD}$ speed in posterior-anterior plane was $885 \pm 135.95 \mathrm{~mm} / \mathrm{s}$, compared to $1020.62 \pm 627 \mathrm{~mm} / \mathrm{s}$ on the external-internal plane. According to these parameters, it seems that the subjects' balance was better in posterior-anterior plane than the external-internal plane.

Investigations disregarded the balance of people with hemipelvectomy amputation. This case report is the first study in this regard. According to Table 4, the Mean \pm SD scores of excursions of center of pressure in the anterior-posterior and external-internal planes were $29 \pm 4$ and $14 \pm 4 \mathrm{~mm}$, respectively [12]. It seems that the study participant has higher relative balance, compared to the normal population. However, the balance was measured during calm stance position, in the present study. The results of the stability analysis in the calm stance position revealed the role of crutch in stability improvement. Considering the lack of muscle support around the hip, knee, and wrist joints, the subject could not have dynamic equilibrium. The alignment of prosthesis components may be another reason for better balance of the subject, compared to the normal population. The alignment of prosthesis components protects the foot in an extra position which stabilizes the person while standing [7]

There are few studies available on gait analysis of the people with hip joint amputation. However, there is no study on the gait analysis of the hemipelvectomy amputees. The obtained results suggested a significant non-symmetry between kinetic and kinematic parameters of the sound and prosthetic sides. Such non-symmetry can be due to muscle weakness and alignment of the prosthesis components. It is recommended that the obtained data of this study be used to design the prosthesis components.

\section{Ethical Considerations}

\section{Compliance with ethical guidelines}

To observe principles of research ethics, an informed consent letter (from the Ethics Committee of Isfahan University of Medical Sciences) was signed by the subject before collecting information. 


\section{Funding}

This research did not receive any specific grant from funding agencies in the public, commercial, or not-forprofit sectors.

\section{Authors contributions}

All authors contributed in preparing this article.

\section{Conflict of interest}

The authors declared no conflict of interest.

\section{References}

[1] Dillingham T, Mackenzie E. Limb amputation and limb deficiency: Epidemiology and recent trends in the United States. Southern Medical Journal. 2002; 95(8):875-83. [DOI:10.1097/00007611-200295080-00019] [PMID]

[2] Bowker JH. Atlas of limb prosthetics: Surgical, prosthetic, and rehabilitation principles. Missouri: Mosby; 2002. [PMM CID]

[3] Unwin N. Epidemiology of lower extremity amputation in centres in Europe, North America and East Asia. British Journal of Surgery. 2000; 87(3):328-37. [DOI:10.1046/j.13652168.2000.01344.x] [PMID]

[4] Denes Z, Till A. Rehabilitation of patients after hip disarticulation. Archives of Orthopedic and Trauma Surgery. 1997; 116(8):498-9. [DOI:10.1007/BF00387586] [PMID]

[5] Zaffer SM, Braddom RL, Conti A, Goff J, Bokma D. Total hip disarticulation prosthesis with suction socket: Report of two cases. American Journal of Physical Medicine \& Rehabilitation / Association of Academic Physiatrists. 1999; 78(2):1602. [DOI:10.1097/00002060-199903000-00017]

[6] Chin T, Kuroda R, Akisue T, Iguchi T, Kurosaka M. Energy consumption during prosthetic walking and physical fitness in older hip disarticulation amputees. Journal of Rehabilitation Research and Development. 2012; 49(8):1255-60. [DOI:10.1682/JRRD.2011.04.0067] [PMID]

[7] Ludwigs E, Bellmann M, Schmalz T, Blumentritt S. Biomechanical differences between two exoprosthetic hip joint systems during level walking. Prosthetics and Orthotics International. 2010; 34(4):449-60. [DOI:10.3109/03093646.2010. 499551] [PMID]

[8] Nietert M, Englisch N, Kreil P, Alba-Lopez G. Loads in hip disarticulation prostheses during normal daily use. Prosthetics and Orthotics International. 1998; 22(3):199-215. [PMID]

[9] Schnall BL, Baum BS, Andrews AM. Gait characteristics of a soldier with a traumatic hip disarticulation. Physical Therapy. 2008; 88(12):1568-77. [DOI:10.2522/ptj.20070337] [PMID]

[10] Yari P, Dijkstra PU, Geertzen JH. Functional outcome of hip disarticulation and hemipelvectomy: A cross-sectional national de- scriptive study in the Netherlands. Clinical Rehabilitation. 2008 22(12):1127-33. [DOI:10.1177/0269215508095088] [PMID]

[11] Kadaba MP, Ramakrishnan HK, Wootten ME, Gainey J, Gorton G, Cochran GV. Repeatability of kinematic, kinetic, and electromyographic data in normal adult gait. Journa of Orthopaedic Research. 1989; 7(6):849-60. [DOI:10.1002/ jor.1100070611] [PMID]

[12] O'Connell M, George K, Stock D. Postural sway and balance testing: A comparison of normal and anterior cruciate ligament deficient knees. Gait \& Posture. 1998; 8(2):136-42. [DOI:10.1016/S0966-6362(98)00023-X] 